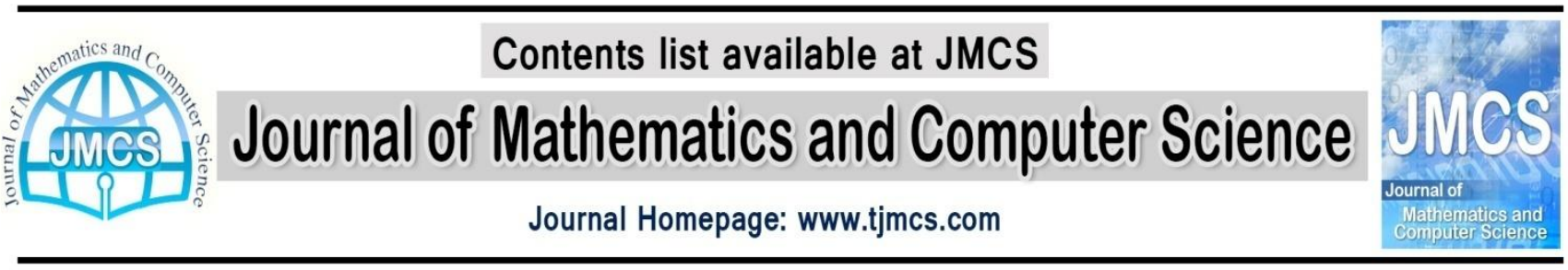

\title{
A New Approach to Solve Fuzzy System of Linear Equations
}

\author{
Sukanta Nayak, S. Chakraverty \\ Department of Mathematics, National Institute of Technology, Rourkela, Odisha -769008, India \\ sukantgacr@gmail.com, \\ sne_chak@yahoo.com
}

Article history:

Received April 2013

Accepted May 2013

Available online May 2013

\begin{abstract}
System of linear equations is a common mathematical phenomenon which occurs in science and engineering problems. But fuzzy systems of linear equations are obtained when we deal with uncertain problems. Various methods are proposed by different authors to solve linear equations for both fuzzy and fully fuzzy systems. Here we have developed an alternative and straight forward arithmetic to handle uncertain interval and fuzzy values. In this arithmetic we write the interval into crisp form by some transformation and used the mathematical limit concept. Fuzzy values are converted into $\alpha$-cut form and then the monotonic functions are operated through the proposed method. Two algorithms have proposed to solve Fully Fuzzy Linear System (FFLS) of equations. Finally some example problems are considered for both fuzzy and fully fuzzy system of linear equations and the obtained results are compared, which are found to be in good argument.
\end{abstract}

Keywords: Uncertainty, Triangular Fuzzy Number (TFN), Trapezoidal Fuzzy Number (TRFN), Fuzzy linear system of equations

\section{Introduction}

Systems of linear equations are frequently found in various field viz. engineering, science and economics etc. As a matter of fact uncertainty plays a vital role in practical case. In this context Zadeh [12] in 1965 had given a novel idea about fuzzy sets for uncertain and imprecise data. Here fuzzy numbers are considered to take care of the uncertainty. For the system of equations $A x=b$ some authors have developed methods by taking (i) only the right hand side column vector as fuzzy, (ii) the coefficient matrix only in fuzzy and (iii) both the coefficient matrix and the right hand side column matrix as fuzzy. Friedman et al. [1] proposed a general method to solve $n \times n$ fuzzy system of linear equations. They took coefficient matrix in crisp and the right hand side vector as fuzzy. Various authors solved fuzzy system of linear equations considering decomposition method. They decomposed the 
coefficient matrix and proposed some new methods. Matinfar et al. [4] used householder decomposition method to solve fuzzy linear equations and they considered only the right hand side column vector as fuzzy and solved some example problems. For the fuzzy coefficient matrix $A$, Panahi et al. [8] obtained lower triangular and upper triangular matrix separately. Then using the modified coefficient matrix $A$, they proposed a new procedure to solve fuzzy system of linear equations. In another paper, Rakhshani [9] used Adomian method for solving the system of fuzzy linear equations. All the above proposed methods are for only Triangular Fuzzy Numbers. Further Nasseri and Gholami [6] took trapezoidal fuzzy number for $n \times n$ fuzzy linear system.

Practically we may not ignore the occurrence of uncertainty in both viz. in coefficient and right hand side vector due to the different types of factors influenced by the system. So there is a need to solve fully fuzzy system of linear equations. In these regard Kumar et al. [2] proposed a new computational method to solve fully fuzzy linear system without any restriction on the coefficient matrix by representing all the parameters as trapezoidal fuzzy numbers. A numerical approximate method has been developed by Liu [3] to solve FFLS. Further he compared the obtained numerical results with Jacobi and Gauss-Seidel methods. Senthilkumar and Rajendran [10] considered symmetric coefficient matrix to solve FFLS. Here they decomposed the coefficient matrix by using Cholesky method. However Vijayalakshmi and Sattanathan [11] introduced ST decomposition procedure to solve FFLS.

Recently a new limit method to handle interval value is presented in Nayak and Chakravrety [7]. This concept has been extended and discussed here for fuzzy system of linear equations. We have modified the traditional interval arithmetic into crisp form. The modified form has been used in a different way to operate fuzzy numbers and proposed a new arithmetic in section 2 . Then we defined triangular and trapezoidal fuzzy number and developed algorithms to solve FFLS in section 3 and 4 respectively. Some example problems are considered and are solved by the proposed method. Obtained results are compared with others and are found to be in good agreement.

\section{Preliminaries}

The uncertain values occurred in practical cases (such as experimental data, impreciseness and partial or imperfect knowledge) may be handled by taking the uncertainty as interval or as fuzzy sense. So to compute these uncertainties we need interval/fuzzy arithmetic. Let us consider the uncertain values in interval form and the same may be written in the following way.

$$
[\underline{x}, \bar{x}]=\{x \mid x \in R, \underline{x} \leq x \leq \bar{x}\}
$$

where $\underline{x}$ and $\bar{x}$ is left and right value of the interval respectively. Let us consider $m=\frac{x+\bar{x}}{2}$ as the mid value or the centre and $w=\bar{x}-\underline{x}$ is the width of the interval $[\underline{x}, \bar{x}]$.

Let us assume that $[\underline{x}, \bar{x}]$ and $[\underline{y}, \bar{y}]$ be two intervals then

1. $[\underline{x}, \bar{x}]+[\underline{y}, \bar{y}]=[\underline{x}+\underline{y}, \bar{x}+\bar{y}]$

2. $[\underline{x}, \bar{x}]-[\underline{y}, \bar{y}]=[\underline{x}-\bar{y}, \bar{x}-\underline{y}]$

3. $[\underline{x}, \bar{x}] \times[\underline{y}, \bar{y}]=[\min \{\underline{x} \underline{y}, \underline{x} \bar{y}, \bar{x} \underline{y}, \bar{x} \bar{y}\}, \max \{\underline{x} \underline{y}, \underline{x} \bar{y}, \bar{x} \underline{y}, \bar{x} \bar{y}\}]$

4. $[\underline{x}, \bar{x}] \div[\underline{y}, \bar{y}]=[\min \{\underline{x} \div \underline{y}, \underline{x} \div \bar{y}, \bar{x} \div \underline{y}, \bar{x} \div \bar{y}\}, \max \{\underline{x} \div \underline{y}, \underline{x} \div \bar{y}, \bar{x} \div \underline{y}, \bar{x} \div \bar{y}\}]$

Now we may extend this concept into various fuzzy numbers viz. triangular and trapezoidal fuzzy numbers etc. We may define any arbitrary fuzzy number in terms of interval involving left and right continuous linear functions. Now fuzzy numbers may be represented as a ordered pair form $[\underline{f}(\alpha), \bar{f}(\alpha)], 0 \leq \alpha \leq 1$ where $\underline{f}(\alpha)$ and $\bar{f}(\alpha)$ are left and right monotonic increasing and decreasing functions over $[0,1]$ respectively.

Let us consider two fuzzy numbers $x=[\underline{x}(\alpha), \bar{x}(\alpha)]$ and $y=[\underline{y}(\alpha), \bar{y}(\alpha)]$ and a scalar $k$ then 
$\begin{array}{ll}\text { i. } & x=y \text { if and only if } \underline{x}(\alpha)=\underline{y}(\alpha) \text { and } \bar{x}(\alpha)=\bar{y}(\alpha) . \\ \text { ii. } & x+y=[\underline{x}(\alpha)+\underline{y}(\alpha), \bar{x}(\alpha)+\bar{y}(\alpha)] . \\ \text { iii. } & k x= \begin{cases}{[k \underline{x}(\alpha), k \bar{x}(\alpha)],} & k \geq 0, \\ {[k \bar{x}(\alpha), k \underline{x}(\alpha)],} & k<0 .\end{cases} \end{array}$

\section{Definition 2.1}

The above discussed interval arithmetic for real interval values have been defined here as follows.

1. $[\underline{x}, \bar{x}]+[\underline{y}, \bar{y}]=\left[\min \left\{\lim _{n \rightarrow \infty} l_{1}+\lim _{n \rightarrow \infty} l_{2}, \lim _{n \rightarrow 1} l_{1}+\lim _{n \rightarrow 1} l_{2}\right\}, \max \left\{\lim _{n \rightarrow \infty} l_{1}+\lim _{n \rightarrow \infty} l_{2}, \lim _{n \rightarrow 1} l_{1}+\lim _{n \rightarrow 1} l_{2}\right\}\right]$

2. $[\underline{x}, \bar{x}]-[\underline{y}, \bar{y}]=\left[\min \left\{\lim _{n \rightarrow \infty} l_{1}-\lim _{n \rightarrow 1} l_{2}, \lim _{n \rightarrow 1} l_{1}-\lim _{n \rightarrow \infty} l_{2}\right\}, \max \left\{\lim _{n \rightarrow \infty} l_{1}-\lim _{n \rightarrow 1} l_{2}, \lim _{n \rightarrow 1} l_{1}-\lim _{n \rightarrow \infty} l_{2}\right\}\right]$

3. $[\underline{x}, \bar{x}] \times[\underline{y}, \bar{y}]=\left[\min \left\{\lim _{n \rightarrow \infty} l_{1} \times \lim _{n \rightarrow \infty} l_{2}, \lim _{n \rightarrow 1} l_{1} \times \lim _{n \rightarrow 1} l_{2}\right\}, \max \left\{\lim _{n \rightarrow \infty} l_{1} \times \lim _{n \rightarrow \infty} l_{2}, \lim _{n \rightarrow 1} l_{1} \times \lim _{n \rightarrow 1} l_{2}\right\}\right]$

4. $[\underline{x}, \bar{x}] \div[\underline{y}, \bar{y}]=\left[\min \left\{\lim _{n \rightarrow \infty} l_{1} \div \lim _{n \rightarrow 1} l_{2}, \lim _{n \rightarrow 1} l_{1} \div \lim _{n \rightarrow \infty} l_{2}\right\}, \max \left\{\lim _{n \rightarrow \infty} l_{1} \div \lim _{n \rightarrow 1} l_{2}, \lim _{n \rightarrow 1} l_{1} \div \lim _{n \rightarrow \infty} l_{2}\right\}\right]$

where for an arbitrary interval $[\underline{a}, \bar{a}]=\left\{\underline{a}+\frac{w}{n}=l \mid \underline{a} \leq l \leq \bar{a}, n \in[1, \infty)\right\}$ and $w$ is the width of the interval.

\section{Definition 2.2}

A fuzzy number $\tilde{A}=\left[a^{L}, a^{N}, a^{R}\right]$ is said to be triangular fuzzy number when the membership function is given by

$$
\mu_{\tilde{A}}(x)= \begin{cases}0, & x \leq a^{L} ; \\ \frac{x-a^{L}}{a^{N}-a^{L}}, & a^{L} \leq x \leq a^{N} ; \\ \frac{a^{R}-x}{a^{R}-a^{N}}, & a^{N} \leq x \leq a^{R} ; \\ 0, & x \geq a^{R} .\end{cases}
$$

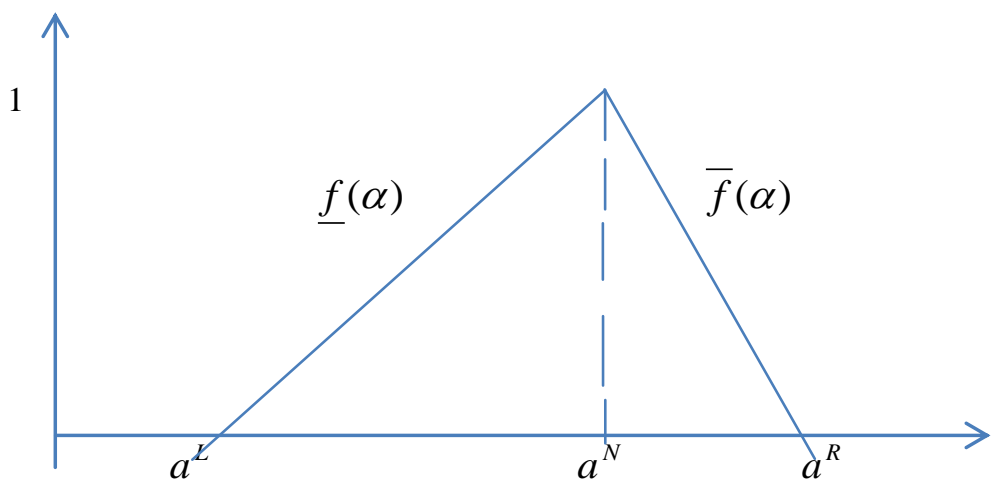

Fig 1. Triangular Fuzzy Number (TFN) 


\section{Definition 2.3}

A fuzzy number $\tilde{A}=\left[a^{L}, a^{N L}, a^{N R}, a^{R}\right]$ is said to be trapezoidal fuzzy number when the membership function is given by

$$
\mu_{\tilde{A}}(x)= \begin{cases}0, & x \leq a^{L} \\ \frac{x-a^{L}}{a^{N L}-a^{L}}, & a^{L} \leq x \leq a^{N L} \\ 1, & a^{N L} \leq x \leq a^{N R} \\ \frac{a^{R}-x}{a^{R}-a^{N R},} & a^{N R} \leq x \leq a^{R} \\ 0, & x \geq a^{R} .\end{cases}
$$

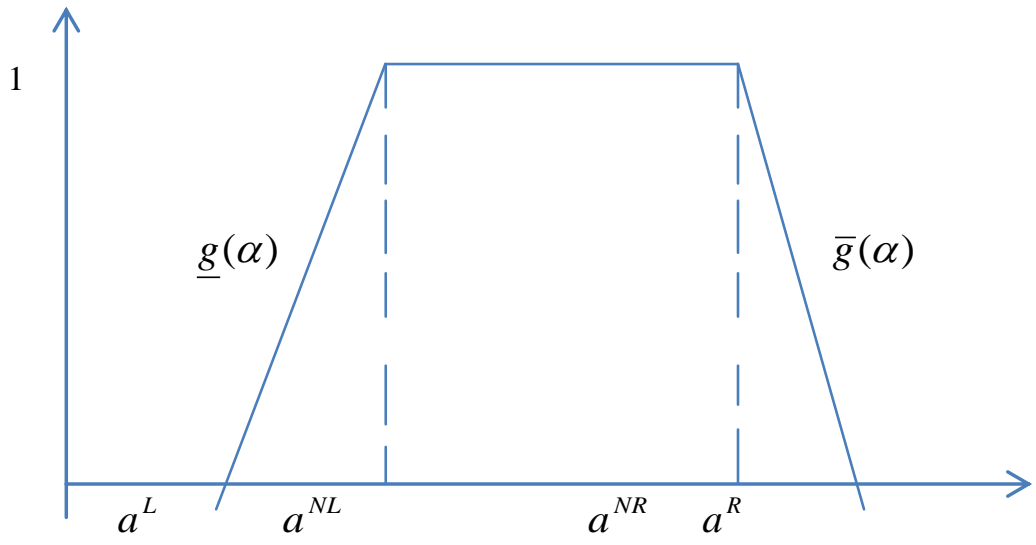

Fig.2 Trapezoidal Fuzzy Number (TRFN)

\section{Definition 2.4}

The Triangular Fuzzy Number $\tilde{A}=\left[a^{L}, a^{N}, a^{R}\right]$ may be transformed into interval form by using $\alpha$-cut as follow.

$$
\tilde{A}=\left[a^{L}, a^{N}, a^{R}\right]=\left[a^{L}+\left(a^{N}-a^{L}\right) \alpha, a^{R}-\left(a^{R}-a^{N}\right) \alpha\right], \quad \alpha \in[0,1] .
$$

Again, the Trapezoidal Fuzzy Number in interval form may also be represented as

$$
\tilde{A}=\left[a^{L}, a^{N L}, a^{N R}, a^{R}\right]=\left[a^{L}+\left(a^{N L}-a^{L}\right), a^{R}-\left(a^{R}-a^{N R}\right)\right], \quad \alpha \in[0,1] .
$$

\section{Definition 2.5}

If the fuzzy numbers are taken in interval form then using definition 2.1 the arithmetic rules may be written as

1. $[\underline{x}(\alpha), \bar{x}(\alpha)]+[y(\alpha), \bar{y}(\alpha)]$

$$
=\left[\min \left\{\lim _{n \rightarrow \infty} m_{1}+\lim _{n \rightarrow \infty} m_{2}, \lim _{n \rightarrow 1} m_{1}+\lim _{n \rightarrow 1} m_{2}\right\}, \max \left\{\lim _{n \rightarrow \infty} m_{1}+\lim _{n \rightarrow \infty} m_{2}, \lim _{n \rightarrow 1} m_{1}+\lim _{n \rightarrow 1} m_{2}\right\}\right]
$$

2. $[\underline{x}(\alpha), \bar{x}(\alpha)]-[\underline{y}(\alpha), \bar{y}(\alpha)]$

$$
=\left[\min \left\{\lim _{n \rightarrow \infty} m_{1}-\lim _{n \rightarrow 1} m_{2}, \lim _{n \rightarrow 1} m_{1}-\lim _{n \rightarrow \infty} m_{2}\right\}, \max \left\{\lim _{n \rightarrow \infty} m_{1}-\lim _{n \rightarrow 1} m_{2}, \lim _{n \rightarrow 1} m_{1}-\lim _{n \rightarrow \infty} m_{2}\right\}\right]
$$

3. $[\underline{x}(\alpha), \bar{x}(\alpha)] \times[\underline{y}(\alpha), \bar{y}(\alpha)]$ 


$$
=\left[\min \left\{\lim _{n \rightarrow \infty} m_{1} \times \lim _{n \rightarrow \infty} m_{2}, \lim _{n \rightarrow 1} m_{1} \times \lim _{n \rightarrow 1} m_{2}\right\}, \max \left\{\lim _{n \rightarrow \infty} m_{1} \times \lim _{n \rightarrow \infty} m_{2}, \lim _{n \rightarrow 1} m_{1} \times \lim _{n \rightarrow 1} m_{2}\right\}\right]
$$

4. $[\underline{x}(\alpha), \bar{x}(\alpha)] \div[\underline{y}(\alpha), \bar{y}(\alpha)]$

$$
=\left[\min \left\{\lim _{n \rightarrow \infty} m_{1} \div \lim _{n \rightarrow 1} m_{2}, \lim _{n \rightarrow 1} m_{1} \div \lim _{n \rightarrow \infty} m_{2}\right\}, \max \left\{\lim _{n \rightarrow \infty} m_{1} \div \lim _{n \rightarrow 1} m_{2}, \lim _{n \rightarrow 1} m_{1} \div \lim _{n \rightarrow \infty} m_{2}\right\}\right]
$$

where for any arbitrary interval

$$
[\underline{f}(\alpha), \bar{f}(\alpha)]=\left\{\underline{f}(\alpha)+\frac{\bar{f}(\alpha)-\underline{f}(\alpha)}{n}=m \mid \underline{f}(\alpha) \leq m \leq \bar{f}(\alpha), n \in[1, \infty)\right\} .
$$

\section{Definition 2.6}

The linear system

$$
\begin{aligned}
& a_{11} x_{1}+a_{12} x_{2}+\ldots+a_{1 n} x_{n}=y_{1} \\
& a_{21} x_{1}+a_{22} x_{2}+\ldots+a_{2 n} x_{n}=y_{2} \\
& \vdots \\
& a_{n 1} x_{1}+a_{n 2} x_{2}+\ldots+a_{n n} x_{n}=y_{n}
\end{aligned}
$$

where the coefficient matrix $A=\left(a_{i j}\right), 1 \leq i \leq n ; 1 \leq j \leq n$ is a $n \times n$ crisp matrix and

$y_{i}, 1 \leq i \leq n$ are fuzzy numbers, is called a fuzzy linear system (FLS). If the coefficient matrix $A=\left(a_{i j}\right)$ and $y_{i}, 1 \leq i \leq n$ both are fuzzy then the system is called fully fuzzy linear system (FFLS).

\section{Linear system of equations with Triangular Fuzzy Numbers}

If the coefficient matrix $A=\left(a_{i j}\right), 1 \leq i \leq n$ and $1 \leq j \leq n$ is a $n \times n$ crisp matrix and $y_{i}, \quad 1 \leq i \leq n$ are TFN, in Eq. (1) a fuzzy linear system (FLS) with TFN whereas if the coefficient matrix $A=\left(a_{i j}\right)$ and $y_{i}, 1 \leq i \leq n$ both are TFN then the system is called FFLS with TFN. Now an algorithm is proposed below to solve linear system of equations with TFN.

\section{Algorithm 1}

Step 1. TFN is written in $\alpha$-cut form.

Let $\left[a^{L}, a^{N}, a^{R}\right]$ be a triangular fuzzy number then it may be represented as $\left[a^{L}, a^{N}, a^{R}\right]=\left[a^{L}+\left(a^{N}-a^{L}\right) \alpha, a^{R}-\left(a^{R}-a^{N}\right) \alpha\right]=[\underline{f}(\alpha), \bar{f}(\alpha)]$.

Step 2. Now the intervals are transferred into the crisp form using the transformation given in definition 2.1.

Step 3. We get a system of linear equations with crisp values. This system may be solved by any standard method used for crisp values.

Step 4. Finally the solution vector would be

$$
x=\left(\left[\lim _{n \rightarrow \infty} x_{1}(\alpha), \lim _{n \rightarrow 1} x_{1}(\alpha)\right],\left[\lim _{n \rightarrow \infty} x_{2}(\alpha), \lim _{n \rightarrow 1} x_{2}(\alpha)\right], \ldots,\left[\lim _{n \rightarrow \infty} x_{n}(\alpha), \lim _{n \rightarrow 1} x_{n}(\alpha)\right]\right)^{T} .
$$

\section{Example 3.1[4]}

Let us consider the following TFN system of linear equations 


$$
\begin{aligned}
& 2 x_{1}+x_{2}+3 x_{3}=[11,19,27] \\
& 4 x_{1}+x_{2}-x_{3}=[-23,-13,-5] \\
& -x_{1}+3 x_{2}+x_{3}=[10,15,27]
\end{aligned}
$$

The above Eq. (2) may be transformed into the following interval form

$2 x_{1}+x_{2}+3 x_{3}=[11+8 \alpha, 27-8 \alpha]$

$4 x_{1}+x_{2}-x_{3}=[-23+10 \alpha,-5-8 \alpha]$

$-x_{1}+3 x_{2}+x_{3}=[10+5 \alpha, 27-12 \alpha]$

Applying definition 2.2 the above equations may be transformed into

$$
\begin{aligned}
& 2 x_{1}+x_{2}+3 x_{3}=\left[\lim _{n \rightarrow \infty}\left\{(11+8 \alpha)+\frac{16-16 r}{n}\right\}, \lim _{n \rightarrow 1}\left\{(11+8 \alpha)+\frac{16-16 r}{n}\right\}\right] \\
& 4 x_{1}+x_{2}-x_{3}=\left[\lim _{n \rightarrow \infty}\left\{(-23+10 \alpha)+\frac{18-18 r}{n}\right\}, \lim _{n \rightarrow 1}\left\{(-23+10 \alpha)+\frac{18-18 r}{n}\right\}\right] \\
& -x_{1}+3 x_{2}+x_{3}=\left[\lim _{n \rightarrow \infty}\left\{(10+5 \alpha)+\frac{17-17 r}{n}\right\}, \lim _{n \rightarrow 1}\left\{(10+5 \alpha)+\frac{17-17 r}{n}\right\}\right]
\end{aligned}
$$

Now solving Eq. (4) we may get the solution vector as

$$
x=\left(\left[-\frac{45}{11}+\frac{23}{11} \alpha,-\frac{10}{11}-\frac{12}{11} \alpha\right],\left[-\frac{2}{11}+\frac{4}{11} \alpha, \frac{68}{11}-\frac{46}{11} \alpha\right],\left[\frac{71}{11}+\frac{6}{11} \alpha, \frac{83}{11}-\frac{6}{11} \alpha\right]\right)^{T}
$$

The obtained results are compared with the results of reference [4] for different membership functions. The results are presented in Table 1.

Table1. Comparison between the solutions

\begin{tabular}{|c|c|c|c|c|}
\hline \multirow{2}{*}{$x$} & \multicolumn{2}{|c|}{ Proposed method } & \multicolumn{2}{c|}{ Ref. [4] } \\
\cline { 2 - 5 } & Left & Right & Left & Right \\
\hline$x_{1}$ & $-\frac{45}{11}+\frac{23}{11} \alpha$ & $-\frac{10}{11}-\frac{12}{11} \alpha$ & $-4+2 \alpha$ & $-1-\alpha$ \\
\hline$x_{2}$ & $-\frac{2}{11}+\frac{4}{11} \alpha$ & $\frac{68}{11}-\frac{46}{11} \alpha$ & $1+\alpha$ & $5-3 \alpha$ \\
\hline$x_{3}$ & $\frac{71}{11}+\frac{6}{11} \alpha$ & $\frac{83}{11}-\frac{6}{11} \alpha$ & $6+\alpha$ & $8-\alpha$ \\
\hline
\end{tabular}

\section{Example 3.2}

Next let us consider the fully fuzzy system of linear equations $A x=b$ where $A=\left[\begin{array}{ccc}{[5,6,10]} & {[3,5,7]} & {[1,3,4]} \\ {[4,12,32]} & {[2,14,29]} & {[0,8,18]} \\ {[14,24,58]} & {[2,32,62]} & {[1,20,44]}\end{array}\right]$ and $b=\left[\begin{array}{c}{[1,2,3]} \\ {[0,1,2]} \\ {[3,5,7]}\end{array}\right]$.

The matrix $A$ and vector $b$ may be transformed into interval form using the above algorithm 1 and accordingly we get 


$$
A=\left[\begin{array}{ccc}
{[5+\alpha, 10-4 \alpha]} & {[3+2 \alpha, 7-2 \alpha]} & {[1+2 \alpha, 4-\alpha]} \\
{[4+8 \alpha, 32-20 \alpha]} & {[2+12 \alpha, 29-15 \alpha]} & {[8 \alpha, 18-10 \alpha]} \\
{[14+10 \alpha, 58-34 \alpha]} & {[2+30 \alpha, 62-30 \alpha]} & {[1+19 \alpha, 44-24 \alpha]}
\end{array}\right] \text { and } b=\left[\begin{array}{c}
{[1+\alpha, 3-\alpha]} \\
{[\alpha, 2-\alpha]} \\
{[3+2 \alpha, 7-2 \alpha]}
\end{array}\right]
$$

Finally the solution vector is obtained as

$$
x=([0.1818,0.7083,1.6538],[-4,-2.25,-0.3636,],[1.1818,3,3.6154])^{T} .
$$

\section{Linear system of equations with Trapezoidal Fuzzy Numbers}

The above Eq. (1) with TRFN may be converted into left monotonically increasing and right monotonically decreasing continuous functions over $[0,1]$. Then it may be solved by the following algorithm.

\section{Algorithm 2}

Step 1. Convert the TRFN into the following form

$$
\left[a^{L}, a^{N L}, a^{N R}, a^{R}\right]=[g(\alpha), \bar{g}(\alpha)] \text {. }
$$

Step 2. Now apply definition 2.1 .

Step 3. We get a system of linear equations is obtained with crisp values. This system may be solved by any standard method used for crisp values.

Step 4. Finally the solution vector would be

$$
x=\left(\left[\lim _{n \rightarrow \infty} x_{1}(\alpha), \lim _{n \rightarrow 1} x_{1}(\alpha)\right],\left[\lim _{n \rightarrow \infty} x_{2}(\alpha), \lim _{n \rightarrow 1} x_{2}(\alpha)\right], \ldots,\left[\lim _{n \rightarrow \infty} x_{n}(\alpha), \lim _{n \rightarrow 1} x_{n}(\alpha)\right]\right)^{T} .
$$

\section{Example 4.1[5]}

Here we consider the following system of linear equations with trapezoidal fuzzy number

$$
\begin{aligned}
& x_{1}-x_{2}=[-31,-1,3,30] \\
& x_{1}+5 x_{2}=[-65,1,13,100]
\end{aligned}
$$

The above Eq. (5) may be transferred into following $\alpha$ - cut form

$$
\begin{aligned}
& x_{1}-x_{2}=[-31+30 \alpha, 30-27 \alpha] \\
& x_{1}+5 x_{2}=[-65+66 \alpha, 100-87 \alpha]
\end{aligned}
$$

Using algorithm 2 we get the solution as

$$
x=([-36.6667,-0.6667,4.6667,41.6667],[-5.6667,0.333,1.6667,11.6667])^{T} .
$$

\section{Example 4.2[2]}

Finally the fully fuzzy system of linear equations with TRFN as considered

$$
[1,3,6,8] x_{1}+[3,4,6,8] x_{2}=[7,27,66,136]
$$$$
[-5,1,2,4] x_{1}+[2,4,5,7] x_{2}=[-41,17,37,92]
$$

Again with the help of $\alpha-$ cut Eq. (7) becomes,

$$
\begin{aligned}
& {[1+2 \alpha, 8-2 \alpha] x_{1}+[3+\alpha, 8-2 \alpha] x_{2}=[7+20 \alpha, 136-70 \alpha]} \\
& {[-5+6 \alpha, 4-2 \alpha] x_{1}+[2+2 \alpha, 7-2 \alpha] x_{2}=[-41+66 \alpha, 92-55 \alpha]}
\end{aligned}
$$

Finally solving Eq. (8) using algorithm 2, we get the solution vector as $x=([8.0588,5,6,9],[-0.3529,3,5,8])^{T}$. 


\section{Conclusion}

A new representation of interval arithmetic has been presented in this paper. The proposed arithmetic is used to develop algorithms to solve FLS and FFLS with both triangular and trapezoidal type of fuzzy numbers. Some example problems are discussed by using these algorithms. The obtained results are compared and it may be seen that the proposed method is simple and efficient. It may be worth mentioning that the idea presented here may easily be used with other fuzzy numbers also.

\section{Acknowledgement}

The authors would like to thank BRNS (Board of Research in Nuclear Sciences), Department of Atomic Energy, (DAE), Govt. of India for providing fund to do this work.

\section{REFERENCES}

[1] Friedman M., Ming M., Kandel A., Fuzzy Linear Systems, Fuzzy Sets and Systems, 96 201-209(1998).

[2] Kumar Amit, Bansal Abhinav, Neetu, A method for solving fully fuzzy linear system with trapezoidal fuzzy numbers, Iranian Journal of Optimization, 2359-374(2010).

[3] Liu Hsuan-Ku, On the Solution of Fully Fuzzy Linear Systems, International Journal of Computational and Mathematical Sciences 4:1 (2010).

[4] Matinfar M., Nasseri S. H. and Sohrabi M., Solving Fuzzy Linear System of Equations by Using Householder Decomposition Method, Applied Mathematical Sciences, Vol. 2, no. 52, 2569 - 2575(2008).

[5] Nasseri Seyed Hadi, Fuzzy Linear Systems: A Decomposition Method and Some New Results, Journal of Applied Mathematics, Islamic Azad University of Lahijan, Vol.5, No.17, Summer (2008).

[6] Nasseri S.H. and Gholami M., Linear System of Equations with Trapezoidal Fuzzy Numbers, The Journal of Mathematics and Computer Science, Vol. 3 No. 1 71-79(2011).

[7] Nayak S. and Chakraverty S., Fuzzy Finite Element Approach for Solving Uncertain Steady State Heat Conduction Problem, National Conference on Mathematics of Soft Computing (NCMSC-2012), NIT Calicut, India.

[8] Panahi A., Allahviranloo T., Rouhparvar H., Solving Fuzzy Linear Systems of Equations, ROMAI J., 4, 1207214(2008),

[9] Rakhshani R., A Novel Method to Solve Fuzzy Linear Systems, Applied Mathematical Sciences, Vol. 2, no. 1, 47 55(2008).

[10] Senthilkumar P. and Rajendran G., New Approach to Solve Symmetric Fully Fuzzy Linear Systems, Sādhanā, Vol. 36, Part 6, December pp. 933-940(2011).

[11] Vijayalakshmi V. and Sattanathan R., ST Decomposition Method for Solving Fully Fuzzy Linear Systems Using Gauss Jordan for Trapezoidal Fuzzy Matrices, International Mathematical Forum, Vol. 6, no. 45, 2245 - 2254(2011).

[12] Zadeh L.A., Fuzzy Sets, Information and Control, 8 338-353 (1965). 